\section{CALL FOR COORDINATED OFFENSIVE ON OBESITY AND DENTAL DECAY}

The British Society of Paediatric Dentistry (BSPD) is calling for a coordinated offensive to tackle both obesity and dental decay in children and young people. A new position statement from BSPD highlights the diet and lifestyle factors common to obesity and dental decay which are both alarmingly prevalent among under $16 s$.

The most recent (2013/14) National Child Measurement Programme (NCMP) showed that $33 \%$ of 11 -year-olds were overweight or obese and research shows that obese young people are more likely to have decay in their permanent teeth.

BSPD spokeswoman Claire Stevens said: 'Poor nutrition poses a clear health risk to children and young people. We support all calls for clearer food labelling and our members wish to be part of a co-ordinated approach to raising awareness of the damaging impact of foods and drinks

which are high in sugar or fat, or both, and low in vitamins'

The BSPD supports national healthy eating campaigns such as the change4life Sugar swaps and the BDA's Make a meal of it campaign, the Action on Sugar campaign and NHS Choices Eat well plate.

The BSPD's position paper can be found at http://ow.ly/Jz2Hd.

\title{
ORAL SCREENING IS A ROLE FOR DENTAL HYGIENISTS AND THERAPISTS
}

According to a new study dental hygienist and therapists should be used to screen for dental caries and periodontal disease in a bid to improve the delivery of primary dental health care.

The findings of the report - The Efficacy of Screening for Common Dental Diseases by Hygiene-Therapists A Diagnostic Test Accuracy Study ${ }^{1}$ - has important ramifications for service design in public-funded health systems.

Lead author, Richard Macey, research assistant at Manchester's School of Dentistry, explained: 'Researchers here at the School of

I Dentistry have been examining whether dental care professionals (DCPs) can screen for common oral disorders. The research is part of a five-year programme that is supported by a NIHR Clinician Scientist award granted to Dr Paul Brocklehurst to examine the efficacy and cost-effectiveness of role substitution in primary dental care.

'We now have the results of the main study which recruited 1,899 asymptomatic patients into 10 dental practices across the north west of England. Before their full check-up, patients saw a dental hygienist and therapist (H\&T)

I and a general dental practitioner (GDP) for a

Iscreening assessment. Clinicians were asked to 음 decide whether the patient screened positive I for dental caries or periodontal disease and so required further investigation.

I The results show a comparable performance between H\&Ts and GDPs and demonstrated H\&Ts can screen for the two principal dental diseases.

'In particular, $\mathrm{H} \&$ Ts were good at identifying those patients the GDP had confirmed were caries free (specificity 0.87 ) and at identifying periodontal disease where the dentists confirmed its presence (sensitivity 0.89 ).

Richard Macey believes these findings, coupled with the recent GDC regulatory change permitting direct access to DCPs, opens up the potential for a 'much more comprehensive use of skill mix in the design and delivery of dental services'

Dr Paul Brocklehurst will be presenting the results in full at the BDA Conference \& Exhibition (7-9 May) at the Manchester Central Convention Complex.

1. http://jdr.sagepub.com/content/early/2015/01/19/ 0022034514567335 .abstract
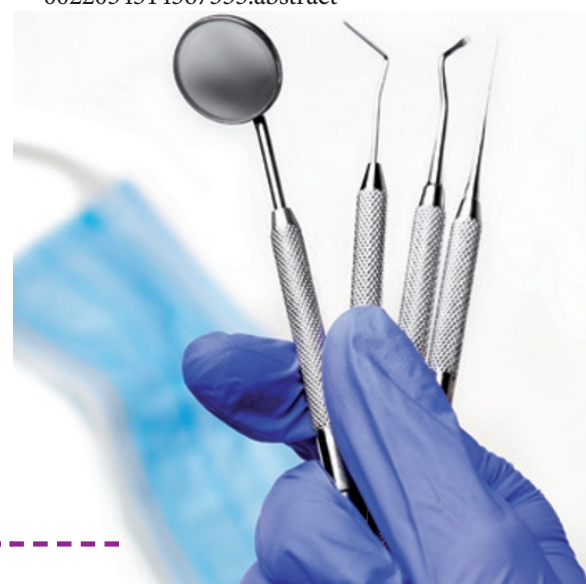

SUGAR CONSUMPTION MUST BE REDUCED THROUGHOUT LIFE

The World Health Organization (WHO) recommends a reduced intake of free sugars throughout life for both adults and children in their new guideline, which is due to be published. Following a consultation that ended in March 2014, the guideline Sugars intake for adults and children recommends that both adults and children reduce their intake of free sugars to less than $10 \%$ of total energy intake.

Free sugars include monosaccharides and disaccharides added to foods and beverages by the manufacturer, cook or consumer, and sugars naturally present in honey, syrups, fruit juices and fruit juice concentrates.

The recommendations are based on evidence reviewed regarding the relationship between free sugars intake and body weight and dental caries. Increasing or decreasing dietary sugars is associated with parallel changes in body weight; the excess body weight associated with free sugars intake results from excess energy intake. The recommendation to limit free sugars intake to less than $10 \%$ of total energy intake is based on evidence from observational studies of dental caries.

The WHO also suggests a further reduction of the intake of free sugars to below $5 \%$ of total energy intake, in recognition that the negative health effects of dental caries are cumulative from childhood to adulthood - therefore even a small reduction in the risk of dental caries in childhood will be of significance in later life.

On the subject of fluoride, the guideline states that although exposure reduces dental caries at a given age and delays the onset of cavities, it does not completely prevent dental caries.

A publication date for Guideline: Sugars intake for adults and children has not yet been announced.
Do you have a news story that you would like included in BDJ Team? Send your press release or a summary of your story to the Editor at bdjteam@nature.com. 